\title{
Pacific
}

Journal of

Mathematics

\section{SMOOTH EXTENSIONS AND QUANTIZED FRÉCHET ALGEBRAS}

XiaOLU WANG 


\title{
SMOOTH EXTENSIONS AND QUANTIZED FRÉCHET ALGEBRAS
}

\section{XiAOLU WANG}

\begin{abstract}
We continue the investigation of the extension theory of smooth algebras in the framework of quantized differential geometry. Here we compute more examples of smooth extensions starting with the cases of dimensions 0 and 1. In particular, we prove a vanishing theorem for totally disconnected spaces. We show that for any compact smooth manifold of positive dimension, there is a representation of $C(M)$ defining a degenerate $\mathcal{L}^{1}$-smooth extension which is not $\mathcal{L}^{1}$-smooth. However for any Fréchet operator ideal $\mathcal{K}_{\tau}$, we prove that all completely positive maps defining extensions of $C^{\infty}\left(S^{1}\right)$ by $\mathcal{K}_{\tau}$ are $\tau$-smooth, and that all the groups $\operatorname{Ext}_{\tau}\left(C^{\infty}\left(S^{1}\right)\right) \simeq \mathbb{Z}$.
\end{abstract}

0. Introduction. This paper is devoted to the study of extension theory of smooth algebras, in the framework of quantized differential geometry. A smooth algebra is a Fréchet *-algebr which is also a dense sublgebra of a $C^{*}$-algebra. A cruxial ingredient is $\tau$ smoothness of a completely positive map. We compute examples of smooth extensions, starting from dimensions 0 and 1 . A vanishing theorem is proved for totally disconnected spaces. For any compact smooth manifold of positive dimension, it is shown that there exists a representation of $C(M)$ which is not $\mathcal{L}^{1}$-smooth as completely positive linear map. However for any Fréchet operator ideal $\mathcal{K}_{\tau}$, we prove that all completely positive maps defining extensions of $C^{\infty}\left(S^{1}\right)$ by $\mathcal{K}_{\tau}$ are $\tau$-smooth, and that all the groups $\operatorname{Ext}_{\tau}\left(C^{\infty}\left(S^{1}\right)\right)$ are isomorphic, although $\operatorname{Ext}_{\tau}\left(C^{\infty}(M)\right)$ varies dramatically depending on the choice of the ideal $\mathcal{K}_{\tau}$, when the dimension of the manifold $M$ is larger than 1.

The theory of $C^{*}$-algbra extensions arose from two origins, one is the work of Brown-Douglas-Fillmore in their classification of essentially normal operators, another is the work of Atiyah and Kasparov on elliptic operators on manifolds. 
Various aspects of smooth extensions have been studied in $[\mathbf{H}-\mathbf{H}]$, $[\mathbf{C - P}],[\mathbf{D}],[\mathbf{D}-\mathbf{V}],[\mathbf{C}],[\mathbf{C}-\mathbf{K}],[\mathbf{S}]$, and $[\mathbf{G}]$ etc. They connect intimately with the index theory, K-theory. It provides a main source of examples and motivations for cyclic homology and noncommutative differential geometry $[\mathbf{C}]$.

While our knowledge of topological Ext-theory is rather complete, a natural and fundamental problem ([A2, p. 9]; [D, p. 68]; [H-H, p. 236]) remains wide open in noncommutative differential geometry, 1.e. the formulation and understanding of the extension theories of smooth algebras.

The notion of $\mathcal{L}^{p}$-smoothness of a B-D-F extension of a manifold was introduced in [D]. It was generalized to normed ideals and $C^{*}$-algebras in $[\mathbf{S l}]$. A vanishing theorem for spheres is proved in [D-V]. For each Fréchet operator ideal $\mathcal{K}_{\tau}$, in [W3] and [W4] we investigated a contravariant bigraded functor $\mathrm{Ext}_{\tau}^{*}$ from the category of smooth algebras to the category of abelian groups, classifying the extensions of a smooth algebra by $\mathcal{K}_{\tau}$. Some features of [W3] distinct from previous works, are that

(1) We worked with all Fréchet operator ideals, instead of Schatten ideals or normed ideals; This is natural in the context of noncommutative differential geometry, because the algebras of infinitely differentiable functions are Fréchet algebras.

(2) We formalized the notion of smooth algebras (c.f. [B-C]), and dealing with classifications of extensions of smooth algebras themselves rather than certain smooth class' in the topological Extgroups of the $C^{*}$-algebras. We must consider not only the restriction of a completely positive map on a set of generators of a $C^{*}$-algebra, but also the continuity of the map with respect to the relevant Fréchet topology.

The dramatic difference due to our new starting point shows immediately on simple examples (see the end of this section).

In [W3] and [W4] we establish a unified framework into which fit all the previous results in this direction. In particular, Voiculescu's theorem and Stinespring's theorem were generalized to smooth algebras and general operator ideals, answering a question raised in [D].

In extending Voiculescu's theorem from algebras having countable bases as vector spaces to Fréchet algebras (c.f. Theorem 1.12 below), 
we were led to the key notion of $\tau$-smoothness of completely positive map, which provides a comparison of the Fréchet topology on a smooth algebra with the topology on the operator ideal $\mathcal{K}_{\tau}$.

As an example of many situations where the notion of $\tau$-smoothness will arise, let us consider the following question. Let $\left(A^{\infty}, A\right)$ be a smooth algebra with a dense subalgebra $A_{0}^{\infty}$. Let

$$
\phi:\left(A^{\infty}, A\right) \longrightarrow \mathcal{L}(\mathfrak{H})
$$

be a completely positive map defining an extension $\varepsilon^{\infty}$ of $A^{\infty}$ by $\mathcal{K}_{\tau}(\mathfrak{H})$, thus also an extension $\varepsilon$ of $A$ by $\mathcal{K}(\mathfrak{H})$.

Suppose that

$$
\phi^{\prime}:\left(A^{\infty}, A\right) \longrightarrow \mathcal{L}(\mathfrak{H})
$$

is another completely positive map, such that $\left.\phi^{\prime}\right|_{A_{0}^{\infty}}$ is (the restriction of) a cross section of $\varepsilon$. Then trivially $\phi^{\prime}$ is a cross section of $\varepsilon$. Now assume that $\left.\phi^{\prime}\right|_{A_{0}^{\infty}}$ is a cross section of $\varepsilon^{\infty}$, must $\left.\phi^{\prime}\right|_{A^{\infty}}$ be a cross section of $\varepsilon^{\infty}$ ?

We show this can be false even for smooth algebras with a single generator (Theorem 2.10). We say that $\phi$ is $\tau$-smooth if this is true for any such $\phi^{\prime}$. A smooth operator algebra is $\tau$-smooth if the identity map is $\tau$-smooth (see 1.10).

Our construction is very general. A group $\operatorname{Ext}_{\tau}\left(A^{\infty}\right)$ is defined for any smooth algebra $A^{\infty}$, even in the extreme case when $A^{\infty}$ is taken to be the $C^{*}$-algebra $A$ itself. Then the functor $\mathrm{Ext}_{\tau}$ is reduced to the B-D-F functor Ext if $\mathcal{K}_{\tau}=\mathcal{K}$. Theorem 2.10 just indicates that when $\mathcal{K}_{\tau} \subsetneq \mathcal{K}$ is a genuine smooth operator ideal, one ought to apply $\mathrm{Ext}_{\tau}$ to "compatible" genuine smooth algebras. It shows that $\operatorname{Ext}_{\mathcal{L}^{1}}$ is not a compatible functor for $C^{*}$-algebras themselves. With modifications, our construction of the functors Ext $_{\tau}$ can be applied to such $\mathcal{K}_{\tau}$ which is a nonideal completion of the algebra $M_{\infty}(\mathbb{C})$ of all the finite matrixes (see 2.22).

From either the point of view of quantized functional analysis $[\mathbf{E}]$, or the point of view of quantization [W4], $\tau$-smooth operator algebras can be considered as quantized Fréchet algebras of various degrees of smoothness.

In [W4], starting with classical examples, we will reformulate the theory of the smooth extensions as a generalization of the scheme of quantizations of Poisson algebras. This establishes a direct con- 
nection between quantum geometry and quantum physics, parallel to the approach of deformation quantization taken e.g. in $[\mathbf{R}]$.

In this paper we derive several useful properties of the smooth Ext $_{\tau}$-theory, and illustrate by more examples. We prove, among other things, that $\mathrm{Ext}_{\tau}$ is always an additive functor (Theorem 2.21) from smooth algebras to abelian groups. In [W3] it is shown that the Toeplitz extension given by the unilateral shift is $\mathcal{L}^{1}$-smooth. Using somewhat different techniques here we show that all completely positive maps defining extensions of $C^{\infty}\left(S^{1}\right)$ by $\mathcal{K}_{\tau}$ are $\mathcal{K}_{\tau^{-}}$ smooth for any Fréchet operator ideal $\mathcal{K}_{\tau}$ (Theorem 3.7).

This is the first of a series of computations of examples and applications of Ext $\tau_{-}$-groups ([W4], [W5], [W7], [W8], [W9]). We start from spaces of dimensions 0 and 1 . We prove that the groups Ext $_{\tau}$ vanish for reasonable smooth algebras on all totally disconnected spaces (Corollary 3.4). We show that $\operatorname{Ext}_{\tau} C^{\infty}\left(S^{1}\right)$ are all isomorphic (Theorem 3.8) for any Fréchet operator ideals. An analogous result for the group $S m$ Ext introduced by Salinas has been obtained in [Sl], where the connection between quasitriangularity and smooth extensions is also studied.

This isomorphism theorem holds only for $S^{1}$. In [W7], we proved that if $n>1$ the group $\operatorname{Ext}_{\mathcal{L}^{1}} C^{\infty}\left(S^{2 n-1}\right)$ is enoumous: it contains all dimension one closed de Rham currents in $S^{2 n-1}$ ! This is in sharply contrast to the well-known vanishing theorem [D-V] that any $\mathcal{L}^{n-1}$-smooth $\left(\mathcal{L}^{2 n-2}\right.$-smooth in our convention) extension of $C^{\infty}\left(S^{2 n-1}\right)$ is trivial (in $\left.\operatorname{Ext} S^{2 n-1}\right)$.

A $K$-theory of Fréchet algebra has been studied by N.C. Phillips [P] (also see [W2]). For manifolds topological $K$-homology is much more difficult to formulate than the topological $K$-theory, although they are isomorphic to each other, via the pairing. It is reasonable to expect that the smooth $K$-cohomology groups as formulated in $[\mathbf{P}]$ and [W2] are independent of the choice of the corresponding smooth operator ideals $\mathcal{K}_{\tau}$, however the smooth $K$-homology group $\operatorname{Ext}_{\tau}\left(C^{\infty}(M)\right)$ varies dramatically depending on the dimension of $M$ and the size of $\mathcal{K}_{\tau}$, as we shall see. Smooth $K$-homology for manifolds has always been a mystery. Now it is seems to be within reach [W7].

Although this paper is a continuation of [W3], the crucial notions are briefly discussed in $\S 1$ to make it reasonably self-contained. 
We shall use the notation in [W3]. Let $\mathfrak{H}$ be a separable Hilbert space. We denote by $\mathcal{L}(\mathfrak{H}), \mathcal{K}(\mathfrak{H})$, and $\mathcal{K}_{f}(\mathfrak{H})$ (or simply $\mathcal{L}, \mathcal{K}, \mathcal{K}_{f}$ ) respectively the algebras of all bounded, compact, and finite rank operators in $\mathfrak{H}$. By $C(X)$ we denote the $C^{*}$-algebra of complex continuous functions on a compact space $X$.

\section{Smooth Algebras, $\tau$-Quasicentrality, and $\tau$-smoothness.}

1.1. By a Fréchet algebra, we mean a complete topological algebra with a countable defining family of submultiplicative seminorms. A smooth algebra is a Fréchet *-algebra $A^{\infty}$ with a specified norm $p$ such that $p\left(a^{*} a\right)=p(a)^{2}$ for all $a \in A^{\infty}$. Often it is written as a pair $\left(A^{\infty}, A\right)$; where $A$ is the $C^{*}$-completion of $A^{\infty}$ with respect to the specified norm $p$, containing the Fréchet ${ }^{*}$-algebra $A^{\infty}$ as a dense *-subalgebra.

The prototype examples of smooth algebras are of course, $\left(C^{\infty}(M), C(M)\right)$, for a smooth compact manifold $M$, where $C(M)$ is the $C^{*}$-algebra of complex continuous functions and $C^{\infty}(M)$ is the subalgebra of all infinitely differentiable functions. While a $C^{*}$-algebra retains only the topological structure of the "space", a smooth algebra is encoded the "smooth" structure of the "manifold". Depending on the chosen smooth subalgebra, the "smooth structure" can be of "smoothness" of various degrees for a smooth manifold, an analytic structure for an analytical manifold, or a PLstructure for a piecewise linear manifold, etc.

The smooth category is the category of all separable smooth algebras. A morphism

$$
f^{\infty}:\left(A^{\infty}, p\right) \longrightarrow\left(B^{\infty}, q\right)
$$

of smooth algebras is a morphism $f^{\infty}$ of Fréchet *-algebras which is contractive with respect to the seminorms $p$ and $q$.

1.2. In general, given a Fréchet algebra $A^{\infty}$, one can get many $C^{*}$-completions $A$ of $A^{\infty}$ by assigning inequivalent $C^{*}$-norms. For example, let $A^{\infty}=L^{1}(G)$ for any nonamenable group $G$, such as the free group on two generators. Then the reduced group $C^{*}$-algebra $C_{r}^{*}(G)$ of $G$ is different from the full group $C^{*}$-algebra $C^{*}(G)$.

On the other hand, the smooth algebra $C^{\infty}(M)$ determines the commutative $C^{*}$-algebra $C(M)$ for a compact smooth manifold $M$. 
This is also true for many interesting noncommutative smooth algebras.

Assume that $A^{\infty}$ is a dense subalgebra of a Fréchet algebra $A$, both have the same unit. We say $A^{\infty}$ is stable under holomorphic functional calculus in $A$ if for any $n \in \mathbb{N}$ and $a \in M_{n}\left(A^{\infty}\right)$ one has $f(a) \in M_{n}\left(A^{\infty}\right)$ for any function $f$ holomorphic in a neighborhood of the spectrum of $a$ in $M_{n}(A)$. If $A$ is a Banach algebra and the group of invertible elements is open in $A$, then it is not necessary to go up to the matrixes over $A[\mathbf{S w}]$.

As a Fréchet algebra, $A^{\infty}$ is always closed under holomorphic functional calculus in itself. If $A^{\infty}$ is stable under holomorphic functional calculus in a $C^{*}$-algebra $A$, then $A^{\infty}$ admits a unique $C^{*}$-norm. Indeed any $C^{*}$-norm of $a \in A^{\infty}$ is the spectral radius of $a^{*} a$ in $A^{\infty}$, hence uniquely determined $[\mathbf{C}]$. If $G$ is nonamenable, then $C^{*}(G)$ does not have any Fréchet subalgebra $A^{\infty}$ stable under holomorphic functional calculus in any of its $C^{*}$-algebra closure.

1.3. Let $C_{f}(\mathbb{Z})$ be the algebra of all ("one-sided") finite sequences over $\mathbb{C}$. A seminorm $p$ on $C_{f}(\mathbb{Z})$ is symmetric if for any sequences $s$ and $s^{\prime}$ which differ only by a permutation of entries, then $p(s)=$ $p\left(s^{\prime}\right)$. A locally m-convex topology $\tau$ on $C_{f}(\mathbb{Z})$ is symmetric, if it is defined by a family $\mathcal{P}$ of submultiplicative symmetric seminorms.

Denote by $C_{\tau}^{0}(\mathbb{Z})$ the completion of $C_{f}(\mathbb{Z})$ with respect to a locally m-convex topology $\tau$, given by a defining family $\mathcal{P}$ of submultiplicative symmetric seminorms.

Any compact operator $T \in \mathcal{K}(\mathfrak{H})$ has a canonical expansion in terms of the so called singular numbers

$$
T=\sum s_{n}(T)<e_{n}, \cdot>f_{n} .
$$

where $s_{1}(T) \geq s_{2}(T) \geq \cdots \geq 0$, both $\left\{e_{n}\right\}$ and $\left\{f_{n}\right\}$ form orthonormal bases of $\mathfrak{H}$.

1.4. (cf. Theorem 10.11, [W2]) Let $\tau$ be a locally m-convex symmetric topology on $C_{f}(\mathbb{Z})$. Then

$$
\mathcal{K}_{\tau}(\mathfrak{H})=\left\{T \in \mathcal{K}(\mathfrak{H}) \mid\left(s_{n}(T)\right) \in C_{\tau}^{0}(\mathbb{Z})\right\}
$$

is a proper ideal of $\mathcal{L}(\mathfrak{H})$, which is the completion of $\mathcal{K}_{f}(\mathfrak{H})$ with respect to a locally $\mathrm{m}$-convex topology. 
Conversely, given such an ideal $\mathcal{K}_{\tau}(\mathfrak{H})$, the linear subspace

$$
S=\left\{s=\left(s_{n}\right) \mid \sum<e_{n}, \cdot>e_{n} \in \mathcal{K}_{\tau}(\mathfrak{H})\right\}
$$

has form $C_{\tau}^{0}(Z)$ for a locally m-convex symmetric topology $\tau$ on $C_{f}(\mathbb{Z})$.

The correspondence is bijective. We shall call $\mathcal{K}_{\tau}(\mathfrak{H})$ the ideal of $\tau$-compact operators in $\mathfrak{H}$.

1.5. Assume that $\mathcal{K}_{\tau}$ is defined by a family $\left\{p_{m}\right\}$ of seminorms. We define (not necessarily submultiplicative) seminorms

$$
p_{m}^{\frac{1}{2}}(T):=p_{m}\left(s_{1}(T)^{\frac{1}{2}}, s_{2}(T)^{\frac{1}{2}}, \ldots\right)^{2},
$$

The completion $\mathcal{K}_{\tau}^{2}(\mathfrak{H})$ of $\mathcal{K}_{f}(\mathfrak{H})$ with respect to $\left\{p_{m}^{\frac{1}{2}}\right\}$ is a complete topological ideal of $\mathcal{L}(\mathfrak{H})$.

Similarly we define a locally convex operator ideal $\mathcal{K}_{\tau}^{\frac{1}{2}}(\mathfrak{H})$ to be the completion of $\mathcal{K}_{f}(\mathfrak{H})$ with respect to $\left\{p_{m}^{2}\right\}$, where

$$
p_{m}^{2}(T)=p_{m}\left(s_{n}(T)^{2}\right)^{\frac{1}{2}} .
$$

If $S, T \in \mathcal{K}_{\tau}^{\frac{1}{2}}$, then $S T \in \mathcal{K}_{\tau}$, and we have the Schwarz inequality

$$
p_{m}(S T) \leq\left(p_{m}^{2}(S) p_{m}^{2}(T)\right)^{\frac{1}{2}} .
$$

For example, if $\mathcal{K}_{\tau}=\mathcal{L}^{p}$, for $p \geq 1$, then $\mathcal{K}_{\tau}^{\frac{1}{2}}=\mathcal{L}^{2 p}$ and $\mathcal{K}_{\tau}^{2}=\mathcal{L}^{\frac{p}{2}}$, which is not locally convex if $p<2$.

As examples of Fréchet operator ideals which are not Banach operator ideals, we mention the class $\overline{\mathcal{L}}^{p}:=\cap_{r>p} \mathcal{L}^{r}$, for $p \geq 1$. We note that $\overline{\mathcal{L}}^{p}$ strictly contains the ideal

$$
\mathcal{L}^{p+}(\mathfrak{H}):=\left\{T \in \mathcal{K}(\mathfrak{H}) \mid s_{n}(T)=O\left(n^{-1 / p}\right)\right\} .
$$

There are large classes of Fréchet operator algebras which are ideals only for certain $C^{*}$-subalgebras of $\mathcal{L}(\mathfrak{H})$, to which our theory also applies (2.22). 
1.6. A representation (respectively, a completely positive map ) of a smooth algebra $\left(A^{\infty}, A\right)$ in $\mathcal{L}(\mathfrak{H})$ is just a $C^{*}$-algebra representation (respectively, a completely positive map) from $A$ into $\mathcal{L}(\mathfrak{H})$.

A continuous linear map $f:\left(A^{\infty}, A\right) \rightarrow\left(B^{\infty}, B\right)$ is a continuous linear map from $A$ to $B$ which induces a continuous map from $A^{\infty}$ to $B^{\infty}$.

Likewise a (continuous) completely positive map from a smooth algebra $\left(A^{\infty}, A\right)$ into a smooth algebra $\left(B^{\infty}, B\right)$ is just a usual completely positive map of $C^{*}$-algebras from $A$ into $B$ which inducing a continuous Fréchet linear map from $A^{\infty}$ into $B^{\infty}$.

For simplicity, unless otherwise stated, a representation means a nondegenerate representation, and a completely positive map will always assumed to be either unital, or completely contractive with respect to the operator $C^{*}$-norms.

1.7. A separable operator space $V$ is $\tau$-quasicentral, if there is an increasing sequence $\left\{k_{m}\right\}$ in $\mathcal{K}_{f}^{+}$strongly converging to 1 such that

$$
\lim _{m \rightarrow \infty}\left[k_{m}, a\right]=0 \quad \text { in } \mathcal{K}_{\tau} \text {, for every } a \in V .
$$

For any separable Hilbert space $H$, a fundamental neighborhood of 0 in $\mathcal{K}_{\tau}(H)$ has form

$U\left(H ; p_{1}, \epsilon_{1} ; \ldots ; p_{m}, \epsilon_{m}\right):=\left\{T \in \mathcal{K}_{\tau}(H) \mid p_{j}(T)<\epsilon_{j}, j=1, \ldots, m\right\}$.

where $p_{j} \in \mathcal{P}$ (see 1.4), and $\epsilon_{j}>0, i=1, \ldots, m$.

Recall that a sequence $\left\{k_{n}\right\}$ of operators is said to converge to $k$ strongly if $\left\|k_{n} \xi\right\| \rightarrow 0$ for any vector $\xi$.

Proposition 1.8. Let $V$ be an operator space. The following properties are equivalent:

(a) For every $n \in \mathbb{N}$ and any $n$-tuple $\left(a_{1}, \ldots, a_{n}\right)$ in $V$, there is an increasing sequence $\left\{k_{m}\right\}$ in $\mathcal{K}_{f}^{+}, k_{m} \nearrow 1$ strongly such that

$$
\lim _{m \rightarrow \infty}\left[k_{m}, a_{i}\right]=0 \quad \text { in } \mathcal{K}_{\tau}, \text { for } i=1, \ldots, n .
$$

(b) For every $n \in \mathbb{N}$, given a $n$-tuple $\left(a_{1}, \ldots, a_{n}\right)$ in $V$, there is an increasing sequence $\left\{k_{m}\right\}$ in $\mathcal{K}_{f}^{+}, k_{m} \nearrow 1$ strongly such that for any fundamental neighborhood

$$
U:=U\left(H ; p_{1}, \epsilon_{1} ; \ldots ; p_{r}, \epsilon_{r}\right)
$$


there is an integer $N$ such that

$$
\left[k_{m}, a_{i}\right] \in U, \quad \text { for } \forall m>N, \text { and } i=1, \ldots, n \text {. }
$$

(c) For any sequence $\left\{a_{n}\right\}$ in $V$, there is an increasing sequence $\left\{k_{m}\right\}$ in $\mathcal{K}_{f}^{+}, k_{m} \nearrow 1$ strongly such that

$$
\lim _{m \rightarrow \infty}\left[k_{m}, a_{i}\right]=0 \quad \text { in } \mathcal{K}_{\tau}, \text { for } i=1,2, \ldots
$$

Proof. The equivalence of (a) and (b) follows from the definition. It is trivial that $(\mathrm{c}) \Longrightarrow(\mathrm{b})$.

(b) $\Longrightarrow\left(\right.$ c): Let $\left\{U_{n}\right\}$ be a fundamental family of neighborhoods. For each fixed $n$, we may choose an increasing sequence $\left\{k_{m, n}\right\}$ in $\mathcal{K}_{f}^{+}, k_{m, n} \nearrow 1$ strongly as $m \rightarrow \infty$, and $N(n) \rightarrow \infty$ so that

$$
\left[k_{m, n}, a_{i}\right] \in U_{n}, \quad \text { for } \forall m \geq N(n), \text { and } i=1, \ldots, n .
$$

Now we define $k_{n}:=k_{N(n), n}$, for $n=1,2, \ldots$ Replacing $N(n)$ by larger integers if necessary, we can assume that $k_{n} \nearrow 1$. By construction we have

$$
\left[k_{n}, a_{i}\right] \in U_{n}, \quad \text { for all } n>1, i=1, \ldots, n \text {. }
$$

Thus for arbitrary $i,(2)$ holds.

EXAMPLE 1.9. In [V2] Voiculescu has proved among many other deep results that $n$-tuples of commuting Hermitian operators are $\mathcal{L}^{n}$-perturbations of diagonal $n$-tuples of commuting Hermitian operators, for $n \geq 2$. This in particular solved a problem posed by Halmos.

Let $\left(A^{\infty}, A\right)$ be a commutative smooth algebra such that $A=$ $C(X)$, where $X$ is a compact set embedded in $\mathbb{R}^{n}$. A degenerate smooth extensions of $A^{\infty}$ is given by a $n$-tuple of commuting Hermitian operators such that $X$ is their joint spectrum. Thus Voiculescu's result implies that all degenerate smooth extensions of $A^{\infty}$ by $\mathcal{L}^{n}$ are $\mathcal{L}^{n}$-quasicentral.

1.10. Let $\left(A^{\infty}, A\right)$ be a smooth algebra, with a dense *-subalgebra $A_{0}^{\infty}$, which is countably generated as a vector space. A completely 
positive map $\phi$ from $\left(A^{\infty}, A\right)$ into $\mathcal{L}(H)$ is $\tau$-smooth (or $\mathcal{K}_{\tau}$-smooth) with respect to $A_{0}^{\infty}$, if the following property holds:

If $\phi_{0}$ is a completely positive map from $\left(A^{\infty}, A\right)$ into $\mathcal{L}(H)$ (see 1.6) such that

$$
\left(\phi-\phi_{0}\right)\left(A_{0}^{\infty}\right) \subset \mathcal{K}_{\tau}(H)
$$

then

$$
\left(\phi-\phi_{0}\right)\left(A^{\infty}\right) \subset \mathcal{K}_{\tau}(H)
$$

Let $\left(A^{\infty}, A\right)$ be a smooth operator algebra in $\mathcal{L}(\mathfrak{H})$, and let $\phi$ be a completely positive map from $\left(A^{\infty}, A\right)$ into $\mathcal{L}(H)$. We say $\phi$ is $\tau$-smooth $\bmod \mathcal{K}_{\tau}\left(\right.$ or $\mathcal{K}_{\tau}$-smooth $\left.\bmod \mathcal{K}_{\tau}\right)$ with respect to $A_{0}^{\infty}$, if the following property holds:

If $\phi_{0}$ is a completely positive map from $\left(A^{\infty}, A\right)$ into $\mathcal{L}(H)$ such that

$$
\left(\phi-\phi_{0}\right)\left(A_{0}^{\infty}\right) \subset \mathcal{K}_{\tau}(H), \quad \text { and } \quad\left(\phi-\phi_{0}\right)\left(A^{\infty} \cap \mathcal{K}_{\tau}(\mathfrak{H})\right) \subset \mathcal{K}_{\tau}(H)
$$

then

$$
\left(\phi-\phi_{0}\right)\left(A^{\infty}\right) \subset \mathcal{K}_{\tau}(H)
$$

Although the definition of $\tau$-smoothness depends on the fixed subalgebra $A_{0}^{\infty}$, in practice $A^{\infty}$ is always defined as the closure of certain canonical subalgebra $A_{0}^{\infty}$, with countably, even finitely many generators. Thus we do not always mention the subspace $A_{0}^{\infty}$ if it is obvious.

A smooth operator algebra $\left(A^{\infty}, A\right)$ is called $\tau$-smooth (or $\tau$ smooth $\bmod \mathcal{K}_{\tau}$ ), if the map $\operatorname{id}_{A^{\infty}}$ is $\tau$-smooth (respectively, $\tau$ smooth $\bmod \mathcal{K}_{\tau}$ ).

Proposition 1.11. (a) If a completely positive map $\phi$ from $\left(A^{\infty}, A\right) \subset \mathcal{L}(\mathfrak{H})$ into $\mathcal{L}(H)$ is $\tau$-smooth, than it is also $\tau$-smooth $\bmod \mathcal{K}_{\tau}$.

In particular, if a smooth operator algebra $\left(A^{\infty}, A\right)$ is $\tau$-smooth, than it is also $\tau$-smooth $\bmod \mathcal{K}_{\tau}$.

(b) Any completely positive map is $\mathcal{K}$-smooth.

Proof. (a) It follows from the definition.

(b) Because completely positive maps are continuous in $C^{*}$-norms, the continuous linear extension $\left(\phi-\phi_{0}\right): A \rightarrow \mathcal{K}(H)$ always exists. 
The following is a generalized Voiculescu's theorem.

TheOREM 1.12. Let $\left(A^{\infty}, A\right)$ be a separable operator algebra $\tau$ smooth mod $\mathcal{K}_{\tau}$ in $\mathcal{L}(\mathfrak{H})$. Let $\pi$ be a nondegenerate $\tau$-quasicentral representation of $\left(A^{\infty}, A\right)$ into $\mathcal{L}(\mathfrak{H})$ such that $\left.\pi\right|_{A \cap \mathcal{K}(\mathfrak{H})}=0$. Then there are unitaries $U_{n}: \mathfrak{H} \rightarrow \mathfrak{H} \oplus \mathfrak{H}$ such that

$$
\begin{gathered}
\left(U_{n}^{*}(a \oplus \pi(a)) U_{n}-a\right) \in \mathcal{K}(\mathfrak{H}), \\
\lim \left(U_{n}^{*}(a \oplus \pi(a)) U_{n}-a\right)=0 \quad \text { in } \mathcal{K}(\mathfrak{H})
\end{gathered}
$$

for every $a \in A$; and

$\left(U_{n}^{*}(a \oplus \pi(a)) U_{n}-a\right) \in \mathcal{K}_{\tau}(\mathfrak{H})$, for every $a \in A^{\infty}$, $\left(2^{\prime}\right)$

$$
\lim \left(U_{n}^{*}(a \oplus \pi(a)) U_{n}-a\right)=0 \quad \text { in } \mathcal{K}_{\tau}(\mathfrak{H}), \text { for every } a \in A_{0}^{\infty} .
$$

Proof. The proof is the same as that of Theorem 2.11 in [W3]. We need only to notice that if $a \in A \cap \mathcal{K}_{\tau}$, then $\pi(a)=0$, hence $U_{n}^{*}(a \oplus \pi(a)) U_{n} \in \mathcal{K}_{\tau}(\mathfrak{H})$. By the assumption that $\left(A^{\infty}, A\right)$ is $\tau$ smooth $\bmod \mathcal{K}_{\tau}$, from the proven fact that (1) holds for all $a \in A_{0}^{\infty}$ one concludes that $\left(1^{\prime}\right)$ holds for all $a \in A^{\infty}$.

1.13. An obvious question is: assume that $s^{\infty}$ is a $\tau$-quasicentral and $\tau$-smooth extension of $A^{\infty}$, does $\mathcal{K}_{\tau}$ have a $\tau$-quasicentral approximate identity as an ideal of $E^{\infty}$ ? In other words, can a fixed sequence $\left\{k_{m}\right\}$ in (c) of Proposition 1.8 be chosen so that

$$
\lim _{m \rightarrow \infty}\left[k_{m}, a\right]=0 \text { in } \mathcal{K}_{\tau} \text {, for all } a \in E^{\infty} .
$$

This is very likely to be true if $E^{\infty}$ is $\tau$-smooth operator algebra. To render some intuition, for any $k \in \mathcal{K}_{\tau}$, one considers the derivation

$$
\begin{aligned}
D_{k}: E^{\infty} & \longrightarrow \mathcal{K}_{\tau} \\
x & \longrightarrow[k, x] .
\end{aligned}
$$

The question is exactly if there is an increasing sequence $\left\{k_{m}\right\}$ in $\mathcal{K}_{f}^{+}, k_{m} \nearrow 1$ strongly, such that the restriction of the Lie algebra representation

$$
k \longrightarrow D_{k}
$$

to this sequence is continuous. 


\section{Smooth extensions.}

2.1. A $\tau$-almost representation of $\left(A^{\infty}, A\right)$ is a completely positive map

$$
\phi: A \longrightarrow \mathcal{L}(\mathfrak{H})
$$

such that

$$
\phi(a b)-\phi(a) \phi(b) \in \mathcal{K}_{\tau}^{2}(\mathfrak{H}), \quad a, b \in A^{\infty}
$$

Proposition 2.2. If $\phi$ is a $\tau$-almost representation, then

$$
\phi(a)^{*}=\phi\left(a^{*}\right), \quad \forall a \in A
$$

$$
\phi(a b)-\phi(a) \phi(b) \in \mathcal{K}(\mathfrak{H}), \quad a, b \in A
$$

Proof. By Stinespring's theorem, there is a representation $\pi$ of $A$ in $\mathfrak{H}$ and a projection $P$ in $\mathcal{L}(\mathfrak{H})$ such that

$$
\phi(a)=P \pi(a) P, \quad \forall a \in A .
$$

So one has

$$
\phi(a)^{*}=P \pi(a)^{*} P=\phi\left(a^{*}\right) \in \mathcal{K}(\mathfrak{H}), \quad \forall a \in A .
$$

To see (ii), we observe first that a completely positive map $\phi$ is a $\tau$-almost representation if and only if

$$
\phi\left(a^{*} a\right)-\phi\left(a^{*}\right) \phi(a) \in \mathcal{K}_{\tau}^{2}(\mathfrak{H}), \quad \forall a \in A^{\infty} .
$$

In fact replacing $a$ by $a+b$ in (2) we get

$$
\left[\phi\left(a^{*} b\right)-\phi\left(a^{*}\right) \phi(b)\right]+\left[\phi\left(b^{*} a\right)-\phi\left(b^{*}\right) \phi(a)\right] \in \mathcal{K}_{\tau}^{2}(\mathfrak{H}) . \quad \forall a, b \in A^{\infty} .
$$

Replace $b$ by $i b$ in (3),

$$
\left[\phi\left(a^{*} b\right)-\phi\left(a^{*}\right) \phi(b)\right]-\left[\phi\left(b^{*} a\right)-\phi\left(b^{*}\right) \phi(a)\right] \in \mathcal{K}_{\tau}^{2}(\mathfrak{H}), \quad \forall a, b \in A^{\infty} .
$$




\section{Hence}

$$
\left[\phi\left(a^{*} b\right)-\phi\left(a^{*}\right) \phi(b)\right] \in \mathcal{K}_{\tau}^{2}(\mathfrak{H}), \quad \forall a, b \in A^{\infty} .
$$

and we get (1) in 2.1. Now the linear map

$$
a \longrightarrow \phi\left(a^{*} a\right)-\phi\left(a^{*}\right) \phi(a)=P \pi\left(a^{*}\right)(1-P) \pi(a) P \in \mathcal{K}, \quad a \in A^{\infty},
$$

is $C^{*}$-norm continuous, so admits a continuous extension to $A$. Applying the argument above one more time, we get (ii).

From the proceeding proof we have (see [S], Lemma 2.2).

Proposition 2.3. A completely positive map $\phi$ is a $\tau$-almost representation if and only if

$$
\phi\left(a^{*} a\right)-\phi\left(a^{*}\right) \phi(a) \in \mathcal{K}_{\tau}^{2}(\mathfrak{H})
$$

for all $a \in A^{\infty}$.

Given a dense *-subalgebra $A_{0}^{\infty}$ of $A^{\infty}$, in order that a completely positive map $\phi$ be a $\tau$-almost representation it may not be sufficient to assume that (1) holds only for all $a \in A_{0}^{\infty}$. The passage from $A_{0}^{\infty}$ to $A^{\infty}$ involves the condition of $\tau$-smoothness of smooth operator algebras.

The following is our working definition of extensions(c.f. [C-K]). Because $\mathcal{L} / \mathcal{K}_{\tau}$ has no Hausdorff topology if $\mathcal{K}_{\tau} \neq \mathcal{K}$, it is necessary and actually more convenient to abandon the standard approach via the Busby invariant.

Definition 2.4. A $\tau$-smooth extension of $\left(A^{\infty}, A\right)$ is a pair $(\pi, P)$, where $\pi$ is a representation of $\left(A^{\infty}, A\right)$ in a Hilbert space $\mathfrak{H}$, and $P$ is a projection in $\mathfrak{H}$, such that

$$
[\pi(a), P] \in \mathcal{K}_{\tau}(\mathfrak{H}), \quad \forall a \in A^{\infty} .
$$

and

$$
P \pi(A) P \cap \mathcal{K}(\mathfrak{H})=\{0\} .
$$

2.5. Note that (1) implies

$$
[\pi(a), P] \in \mathcal{K}(\mathfrak{H}), \quad \forall a \in A .
$$

Let

$$
\phi(a):=P \pi(a) P, \quad a \in A .
$$

Then

$$
\phi:\left(A^{\infty}, A\right) \longrightarrow \mathcal{L}(\mathfrak{H}) .
$$

is a $\tau$-almost representation. 


\subsection{Set}

$$
E:=\phi(A)+\mathcal{K}(\mathfrak{H})
$$

and

$$
E^{\infty}:=\phi\left(A^{\infty}\right)+\mathcal{K}_{\tau}(\mathfrak{H})
$$

With the operator norm, $E$ is a $C^{*}$-algebra. Endowing $E^{\infty}$ with the locally convex final topology induced by the maps

$$
i: \mathcal{K}_{\tau} \rightarrow E^{\infty}
$$

and $\phi$, we make $E^{\infty}$ a Fréchet *-algebra. Then $\left(E^{\infty}, E\right)$ is a smooth operator algebra (Proposition 3.6, [W3]).

2.7. Notice that as Fréchet spaces, $E^{\infty}$ is the direct sum of $\mathcal{K}_{\tau}(\mathfrak{H})$ and $\phi\left(A^{\infty}\right)$. We have an exact sequence

$$
0 \longrightarrow \mathcal{K}_{\tau} \stackrel{i}{\rightarrow} \longrightarrow E^{\infty} \stackrel{q_{\tau}}{\rightarrow} \longrightarrow A^{\infty} \longrightarrow 0
$$

in the category of Fréchet *-algebras, and an exact sequence

$$
0 \longrightarrow \mathcal{K} \stackrel{i}{\rightarrow} \longrightarrow E \stackrel{q}{\rightarrow} \longrightarrow A \longrightarrow 0
$$

in the category of $C^{*}$-algebras.

If $A_{0}^{\infty}$ is a dense ${ }^{*}$-subalgebra of $A^{\infty}$ with a countable basis as a vector space, then $E_{0}^{\infty}:=\phi\left(A_{0}^{\infty}\right)+\mathcal{K}_{f}$ is a dense subalgebra of $E^{\infty}$ with the same property.

Note that we do not require $\phi$ itself to be $\tau$-quasicentral.

2.8. From Definition 2.4, every degenerate extension

$$
\pi:\left(A^{\infty}, A\right) \longrightarrow \mathcal{L}(\mathfrak{H})
$$

is a $\tau$-smooth extension for any $\mathcal{K}_{\tau}$. However, $\pi$ is $\tau$-smooth as a completely positive map only for those $\mathcal{K}_{\tau}$ whose topologies are no finer than that on $A^{\infty}$. "Finer" topology translates to "smoother" differential structure, in the more familiar language on commutative manifolds. For example, to say the topology on the smooth algebra $C^{\infty}\left(S^{1}\right)$ is finer then that on $C^{(2)}\left(S^{1}\right)$ translates to saying that the algebra $C^{\infty}\left(S^{1}\right)$ is smoother than $C^{(2)}\left(S^{1}\right)$. To convey more intuition about how $\tau$-smoothness of completely positive maps as the measure comparing the smoothness of a Fréchet algebra and the 
smoothness of an operator ideal $\mathcal{K}_{\tau}$, we illustrate with an example of an extreme case.

Lemma 2.9. Let $A=A^{\infty}=C\left(S^{1}\right)$. There is a representation $\pi: A \longrightarrow \mathcal{L}(H)$ defining a degenerate $\tau$-smooth extension which is $\mathcal{K}$-smooth but not $\mathcal{L}^{1}$-smooth as a completely positive map.

Proof. Here obviously $A_{0}^{\infty}$ is the subalgebra of trigonometric polynomials with a single generator $z=e^{2 \pi i \theta}$ (see 1.10).

In view of (b) of Proposition 1.11, we need only to prove that it is not $\mathcal{L}^{1}$-smooth.

We choose $\pi(z)$ to be the bilateral shift $U$ with respect to the standard basis of $H:=L^{2}\left(S^{1}, \mu\right)$.

Take any $f \in C\left(S^{1}\right)$ and let

$$
f=\sum_{n \in \mathbb{Z}} a_{n} z^{n}
$$

be its Fourier series expansion. Then

$$
\pi(f)=\sum_{n \in \mathbb{Z}} a_{n} U^{n}
$$

Let $\left\{e_{n}\right\}$ be the standard basis of the Hilbert space $H$, where $e_{n}=$ $e^{2 \pi n i \theta}$.

Let $U_{0}$ be the weighted bilateral shift on $H$ given by the matrix

$$
U_{0}=\left(\begin{array}{cccccccc}
\ddots & \ddots & & & \vdots & & & \\
\\
0 & z_{2} & & \vdots & & & & \\
& 0 & z_{1} & \vdots & & & & \\
& & 0 & z_{0} & & & & \\
\ldots & \ldots & \ldots & z_{-1} & \ldots & \ldots & \ldots \\
& & & \vdots & 0 & z_{-2} & & \\
& & & \vdots & & 0 & z_{-3} & \\
& & & \vdots & & & 0 & \ddots \\
& & & & & & & \ddots
\end{array}\right)
$$

with respect to the standard basis $\left\{e_{n}\right\}$, where $z_{n}:=\exp \left(2^{-|n|-2} \pi i\right)$, for $n \in \mathbb{Z}$. Then

$$
U-U_{0}=\sum_{n \in \mathbb{Z}}\left(1-z_{n}\right)<e_{n}, .>e_{n+1} .
$$


The singular numbers are

$$
s_{n}\left(U-U_{0}\right)=\left|1-z_{\left[\frac{n}{2}\right]}\right|=O\left(2^{\left[\frac{n}{2}\right]}\right) .
$$

Thus $U-U_{0} \in \mathcal{L}^{1}(H)$. Now define a representation $\pi_{0}$ of $C\left(S^{1}\right)$ by

$$
\pi_{0}(f)=\sum_{n \in \mathbb{Z}} a_{n} U_{0}^{n}
$$

Then $\pi(f)-\pi_{0}(f) \in \mathcal{L}^{1}(H)$, for all $f \in A_{0}^{\infty}$. However consider

$$
\Delta f=\left(\pi-\pi_{0}\right) f=\sum_{n \in \mathbb{Z}} a_{n}\left(U^{n}-U_{0}^{n}\right) .
$$

A calculation shows its $i$ - $j$ entry is

$$
(\Delta f)_{i, j}=a_{i+j}\left(1-z_{1-j} \ldots z_{i}\right) \quad i, j \in \mathbb{Z}
$$

An estimate yields

$$
z_{1-j} \ldots z_{i}=e^{2 \pi i \alpha_{\imath, 3}}
$$

with

$$
\frac{1}{8} \leq \alpha_{i, j}<\frac{1}{2}, \quad \text { for all } i, j>0
$$

It follows that

$$
(\Delta f)_{i, j}=O\left(a_{i+j}\right)
$$

as $|i|,|j| \longrightarrow \infty$.

Recall that the Fourier series define an isomorphism from $C\left(S^{1}\right)$ to the $C^{*}$-algebra $C^{*}(\mathbb{Z})^{+}$, the unitalization of the $C^{*}$-algebra $C^{*}(\mathbb{Z})$, which is strictly larger than $l^{1}(\mathbb{Z}$ ) (There are plenty functions in $C\left(S^{1}\right)$ such that their Fourier series are not absolutely convergent.) Now we choose

$$
\left(a_{n}\right) \in C^{*}(\mathbb{Z}) \backslash l^{1}(\mathbb{Z}),
$$

then $(\Delta f) \notin \mathcal{L}^{1}(H)$.

In fact, by Ky Fan's inequality $[\mathbf{K y}]$, for any finite set $\left\{i_{1}, \ldots, i_{n}\right\}$ of $n$ distinct integers and $\left\{j_{1}, \ldots, j_{n}\right\}$ of $n$ distinct integers, we have

$$
\sum_{k=1}^{n}(\Delta f)_{i_{k}, j_{k}} \leq \sum_{k=1}^{n} s_{k}(\Delta f) .
$$


Thus (2) and (3) imply that $\Delta f \notin \mathcal{L}^{1}(H)$. Therefore $\pi$ is not $\tau$ smooth as a completely positive map.

THEOREM 2.10. Let $M$ be a compact $C^{(1)}$-manifold of dimension $\leq 1$. $A=A^{\infty}=C(M)$. There is a representation $\pi: A \longrightarrow \mathcal{L}(H)$ defining a degenerate $\tau$-smooth extension, which is $\mathcal{K}$-smooth but not $\mathcal{L}^{1}$-smooth as a completely positive map.

Proof. Fix a $C^{(1)}$-embedding $\alpha$ of the circle $S^{1}$ into $M$. Then $\alpha^{*}(f)=f \circ \alpha$ defines a surjective homomorphism $\alpha^{*}$ from $C(M)$ onto $C\left(S^{1}\right)$. We only need to specify a dense subalgebar $A_{0}$ of $C(M)$ which is countablly generated as a vector space, and $\alpha^{*}\left(A_{0}\right)$ is the algebra $\mathbb{C}[z]$ of (trigonometric) polynomials, where $z$ is the standard coordinate on $S^{1}$. Then the conclusion will follow from Lemma 2.9.

In fact let $\pi$ be the representation of $C\left(S^{1}\right)$ as in the proof of Lemma 2.9 and consider the representations $\tilde{\pi}=\alpha^{*} \circ \pi$ and $\tilde{\pi}_{0}=$ $\alpha^{*} \circ \pi_{0}$ of $C(M)$. Then $\tilde{\pi}(f)-\tilde{\pi}_{0}(f) \in \mathcal{L}^{1}(H)$, for all $f \in A_{0}$, but there are some $f \in A$ such that $\tilde{\pi}(f)-\tilde{\pi}_{0}(f) \notin \mathcal{L}^{1}(H)$.

Choose two tubular neighborhood $V \subset U$ of $\alpha\left(S^{1}\right)$ and a coordinate patch $(U, \phi)$ on $M, \phi=\left(\tilde{z}_{1}, \tilde{z}_{2}, \ldots, \tilde{z}_{n}\right)$, such that $z=\alpha \circ \tilde{z}_{1}$, and $\tilde{z}_{2}^{-1}(0) \cap \cdots \cap \tilde{z}_{n}^{-1}(0)=\alpha\left(S^{1}\right)$.

Now let $\lambda$ be a $C^{(1)}$-function on $M$ equal to 1 on $V$ with support contained in $U$. Let $\tilde{z}_{i}(x)=\lambda z_{i}(x)$ if $x \in U$ and 0 otherwise, for $i=1, \ldots, n$. We may extend $\tilde{z}_{1}, \tilde{z}_{2}, \ldots, \tilde{z}_{n}$ to a family of $C^{(1)}$ functions $\left\{\tilde{z}_{1}, \ldots, \tilde{z}_{n}, \ldots, \tilde{z}_{m+n}\right\}$ on $M$ such that $\tilde{z}_{i}$ vanishes on $V$ for all $i=n+1, \ldots, n+m$. Then the dense subalgebra $A_{0}$ is the algebra $\mathbb{C}\left[\tilde{z}_{1}, \ldots, \tilde{z}_{n}, \ldots, \tilde{z}_{m+n}\right]$ of polynomials.

2.11. We say two $\tau$-smooth extensions

$$
\phi_{i}: A^{\infty} \rightarrow \mathcal{L}\left(\mathfrak{H}_{i}\right)
$$

are (unitarily) equivalent, denoted by $\phi_{1} \underset{\tau}{\rightarrow} \simeq \phi_{2}$, if there is a unitary operator $u: \mathfrak{H}_{1} \rightarrow \mathfrak{H}_{2}$ such that

$$
\phi_{1}(a)-u^{*} \phi_{2}(a) u \in \mathcal{K}_{\tau}\left(\mathfrak{H}_{1}\right), \quad \text { for every } a \in A^{\infty} .
$$

This implies automatically

$$
\phi_{1}(a)-u^{*} \phi_{2}(a) u \in \mathcal{K}\left(\mathfrak{H}_{1}\right), \quad \text { for every } a \in A \text {. }
$$


2.12. Let $\mathfrak{E x t}_{\tau}\left(A^{\infty}\right)$ (resp. $\left.\mathfrak{D} \mathfrak{x t}_{\tau}\left(A^{\infty}\right)\right)$ be the unitary equivalence classes of all the (resp. degenerate) $\tau$-smooth extensions of $A^{\infty}$.

Both $\mathfrak{E x t}_{\tau}\left(A^{\infty}\right)$ and $\mathfrak{D} \mathfrak{x t}_{\tau}\left(A^{\infty}\right)$ form semigroups under the following binary operation + :

$$
\left[\phi_{1}+\phi_{2}\right]:=\left[\phi_{1}\right]+\left[\phi_{2}\right]:=\left[\zeta \circ\left(\phi_{1} \oplus \phi_{2}\right)\right]
$$

where

$$
\zeta: M_{2}(\mathcal{L}(\mathfrak{H})) \longrightarrow \mathcal{L}(\mathfrak{H})
$$

is a spatial isomorphism taking $M_{2}\left(\mathcal{K}_{\tau}(\mathfrak{H})\right)$ onto $\mathcal{K}_{\tau}(\mathfrak{H})$. (See [W3], $\S 5$.

Clearly if $\phi_{1}$ is $\tau$-smooth as a completely positive map, and $\phi_{1} \underset{\tau}{\rightarrow} \simeq$ $\phi_{2}$, then $\phi_{2}$ is also $\tau$-smooth as a completely positive map. If both $\phi_{1}$ and $\phi_{2}$ are $\tau$-smooth as a completely positive map, then $\phi_{1}+\phi_{2}$ is also.

We shall denote by $\mathfrak{E x t}_{s, \tau}\left(A^{\infty}\right)$ ( resp. $\mathfrak{D x t}_{s, \tau}\left(A^{\infty}\right)$ ) the submonoids of those consisting of $\tau$-smooth completely positive maps.

Definition 2.13. For any smooth algebra $A^{\infty}$, we let

$$
\operatorname{Ext}_{\tau}\left(A^{\infty}\right)=\operatorname{Ext}_{\tau}\left(A^{\infty}\right) / \underset{\tau}{\rightarrow} \sim
$$

where the equivalence relation $\underset{\tau}{\rightarrow} \sim$ is defined by $\left[\left[\phi_{1}\right]\right] \underset{\tau}{\rightarrow} \sim\left[\left[\phi_{2}\right]\right]$ if

$$
\left[\phi_{1}\right]+\left[\vartheta_{1}\right] \underset{\tau}{\rightarrow} \simeq\left[\phi_{2}\right]+\left[\vartheta_{2}\right]
$$

for some $\left[\vartheta_{1}\right],\left[\vartheta_{2}\right] \in \mathfrak{D x t}_{\tau}\left(A^{\infty}\right)$.

Clearly with the binary operation + , the collection $\operatorname{Ext}_{\tau}\left(A^{\infty}\right)$ form a monoid with the unit given by $[[\vartheta]]$ for any $\vartheta \in \mathfrak{D x t}_{\tau}\left(A^{\infty}\right)$.

Replacing $\mathfrak{E x t}_{\tau}$ by $\mathfrak{E x t}_{s, \tau}$ (resp. $\mathfrak{D} \mathfrak{x t}_{\tau}$ by $\left.\mathfrak{D x t}_{s, \tau}\right)$ in above, we get the submonoid $\operatorname{Ext}_{s, \tau}\left(A^{\infty}\right)$ of those consisting of $\tau$-smooth completely positive maps.

If $\tau$ is finer than $\tau^{\prime}$, a $\tau$-smooth extension $\phi$ is also a $\tau^{\prime}$-smooth extension by Definition 2.4. Thus there is a natural transformation from $\mathfrak{E x t}_{\tau}$ to $\mathfrak{E x t}_{\tau^{\prime}}$.

TheOREM 2.14. (c.f. Theorem 3.11, [W3]). (a) Ext Ex $_{\tau}$ is a contravariant functor from the category of smooth algebras to the category of abelian groups. 
(b) If $\tau$ is finer than $\tau^{\prime}$, there is a natural transformation $\alpha_{\tau, \tau^{\prime}}$ from $\mathrm{Ext}_{\tau}$ to $\mathrm{Ext}_{\tau^{\prime}}$. In particular there is a natural transformation $\alpha_{\tau}$ from Ext $_{\tau}$ to the $B-D-F$ functor Ext for any $\tau$.

(c) If $\tau$ is finer than $\tau^{\prime}$, and $\tau^{\prime}$ is finer than $\tau^{\prime \prime}$, then

$$
\alpha_{\tau^{\prime}, \tau^{\prime \prime}} \circ \alpha_{\tau, \tau^{\prime}}=\alpha_{\tau, \tau^{\prime \prime}}
$$

and

$$
\alpha_{\tau^{\prime}} \circ \alpha_{\tau, \tau^{\prime}}=\alpha_{\tau}
$$

From (c) above we have immediately

Corollary 2.15. Assume that $\tau$ is finer than $\tau^{\prime}$, and $\tau^{\prime}$ is finer than $\tau^{\prime \prime}$. Then for any smooth algebra $\left(A^{\infty}, A\right)$,

(i)

$$
\alpha_{\tau, \tau^{\prime \prime}}\left(A^{\infty}\right): \operatorname{Ext}_{\tau}\left(A^{\infty}\right) \longrightarrow \operatorname{Ext}_{\tau^{\prime \prime}}\left(A^{\infty}\right)
$$

is surjective implies that

$$
\alpha_{\tau^{\prime}, \tau^{\prime \prime}}\left(A^{\infty}\right): \operatorname{Ext}_{\tau^{\prime}}\left(A^{\infty}\right) \longrightarrow \operatorname{Ext}_{\tau^{\prime \prime}}\left(A^{\infty}\right)
$$

is surjective; $\alpha_{\tau, \tau^{\prime \prime}}\left(A^{\infty}\right)$ is injective implies that

$$
\alpha_{\tau, \tau^{\prime}}\left(A^{\infty}\right): \operatorname{Ext}_{\tau}\left(A^{\infty}\right) \longrightarrow \operatorname{Ext}_{\tau^{\prime}}\left(A^{\infty}\right)
$$

is injective.

(ii)

$$
\alpha_{\tau}\left(A^{\infty}\right): \operatorname{Ext}_{\tau}\left(A^{\infty}\right) \longrightarrow \operatorname{Ext}(A)
$$

is surjective implies that

$$
\alpha_{\tau^{\prime}}\left(A^{\infty}\right): \operatorname{Ext}_{\tau^{\prime}}\left(A^{\infty}\right) \longrightarrow \operatorname{Ext}(A)
$$

is surjective; $\alpha_{\tau}\left(A^{\infty}\right)$ is injective implies that $\alpha_{\tau, \tau^{\prime}}\left(A^{\infty}\right)$ is injective.

Proposition 2.16. Let $\phi:\left(A^{\infty}, A\right) \longrightarrow \mathcal{L}(\mathfrak{H})$ be a $\tau$-smooth extension. If $\phi$ is $\tau$-smooth as a completely positive map with respect to $A_{0}^{\infty}$ (see 1.10), then the operator algebra $E^{\infty}$ (see 2.7$)$ is $\tau$-smooth $\bmod \mathcal{K}_{\tau}$ with respect to $E_{0}^{\infty}$.

Proof. Let's assume

$$
\rho:\left(E^{\infty}, E\right) \longrightarrow \mathcal{L}(\mathfrak{H})
$$


be a completely positive map such that $\rho\left(\mathcal{K}_{\tau}(\mathfrak{H})\right) \subset \mathcal{K}_{\tau}(\mathfrak{H})$ and

$$
(\rho-i d) E_{0}^{\infty} \subset \mathcal{K}_{\tau}(\mathfrak{H})
$$

Then

$$
\phi^{\prime}:=\rho \circ \phi:\left(A^{\infty}, A\right) \longrightarrow \mathcal{L}(\mathfrak{H})
$$

is a completely positive map with $\left(\phi^{\prime}-\phi\right) A_{0}^{\infty} \subset \mathcal{K}_{\tau}(\mathfrak{H})$.

Because $\phi$ is $\tau$-smooth, we have $\left(\phi^{\prime}-\phi\right) A_{0}^{\infty} \subset \mathcal{K}_{\tau}(\mathfrak{H})$, hence

$$
(\rho-i d) \phi\left(A^{\infty}\right) \subset \mathcal{K}_{\tau}(\mathfrak{H})
$$

and

$$
(\rho-i d) E^{\infty}=(\rho-i d)\left(\phi\left(A^{\infty}\right)+\mathcal{K}_{\tau}(H)\right) \subset \mathcal{K}_{\tau}(\mathfrak{H})
$$

2.3. We say $[\phi]$ is absorbing, if

$$
[\phi]+[\vartheta]=[\phi] \quad \text { for any } \vartheta \in \mathfrak{D x t}_{\tau}\left(A^{\infty}\right) .
$$

Denote by $\mathfrak{D x t}_{a, \tau}, \mathfrak{E x t}_{a, \tau}\left(\simeq \operatorname{Ext}_{a, \tau}\right)$ the subgroups consisting of absorbing extensions.

As a consequence of Theorem 1.12, we have

Theorem 2.18 ([W3], Corollary 3.17). If all degenerate extensions in $\mathfrak{D x t}_{\tau}\left(A^{\infty}\right)$ are $\tau$-quasicentral, and at least one of them is $\tau$-smooth, then there is an natural isomorphism of abelian groups

$$
\operatorname{Ext}_{\tau}\left(A^{\infty}\right) \simeq \operatorname{Ext}_{a, \tau}\left(A^{\infty}\right)
$$

LeMma 2.19. Let $X$ be a self-adjoint operator in $\mathcal{L}(\mathfrak{H})$ such that

$$
P(X)=T \in \mathcal{K}_{\tau}(\mathfrak{H}),
$$

for some polynomial $P$ with complex coefficiences. Then $X$ is diagonalizable, i.e. $X$ is unitarily conjugate to a diagonal operator.

Proof. Replacing $P$ by $P+\bar{P}$, we can assume that

$$
P(X)=X^{n}+a_{1} X^{n-1}+a_{2} X^{n-2}+\cdots+a_{n}
$$


where $a_{i}$ are real and the operator $T$ is self-adjoint.

Let $V_{\lambda}$ be the eigenspace of any eigenvalue $\lambda$ of $T$. Denote by

$$
\mathfrak{H}_{\lambda}=\sum_{j=0}^{n-1} X^{j} V_{\lambda}
$$

the corresponding "root space" of $X$. Using (1), one verifies that $\mathfrak{H}_{\lambda}$ is an invariant subspace of $X$. Because $X$ is self-adjoint and $\mathfrak{H}_{\lambda}$ is finite dimensional, $\mathfrak{H}_{\lambda}$ is spanned by the eigenvectors of $X$. Since $T$ is diagonizable, $\mathfrak{H}$ is spanned by all such $V_{\lambda}$, therefore the eigenvectors of $X$ spans $\mathfrak{H}$.

Lemma 2.20. Suppose that $X \in \mathcal{L}(\mathfrak{H})$ satisfies the conditions

$$
X-X^{*} \in \mathcal{K}_{\tau}, \quad X^{2}-X \in \mathcal{K}_{\tau} .
$$

Then there is a $\mathcal{K}_{\tau}$-perturbation $P$ of $X$ which is a projection.

Proof. Replacing $X$ by $\frac{1}{2}\left(X+X^{*}\right)$, we may assume $X$ is already self-adjoint. By assumption

$$
T:=X^{2}-X \in \mathcal{K}_{\tau}(\mathfrak{H}) .
$$

If $V_{\mu}$ is the eigenspace of eigenvalue $\mu$ of $X$, then

$$
\mu^{2}-\mu=\lambda
$$

is an eigenvalue of $T$, and $V_{\mu}$ is contained in the eigenspace of $\lambda$ of $T$. Thus $V_{\mu}$ is finite dimensional.

If $X$ is diagonalizable, then the conclusion follows easily.

By Lemma 2.19, $X$ itself is diagonalizable. (It follows from von Neumann's theorem that a $\mathcal{K}_{\tau}$-perturbation of $X$ is diagonalizable, if $\mathcal{K}_{\tau}(\mathfrak{H}) \supset \mathcal{L}^{2}(\mathfrak{H})$. But this is not good enough for our purpose.) Hence $X$ has a canonical expansion

$$
X=\sum \mu_{n}<v_{n}, \cdot>v_{n}
$$

such that

$$
T=\sum \lambda_{n}<v_{n}, \cdot>v_{n},
$$

with $\lambda_{n}=\mu_{n}^{2}-\mu_{n} \in \sigma(T)$. Let

$$
N_{0}=\left\{n|\quad| \lambda_{n} \mid \geq \frac{1}{4}\right\}
$$




$$
N_{+}=\left\{n \mid \quad \mu_{n}=\frac{1}{2}+\sqrt{\frac{1}{4}-\lambda}, \quad \text { and }\left|\lambda_{n}\right|<\frac{1}{4}\right\} \text {, }
$$

and

$$
N_{-}=\left\{n \mid \quad \mu_{n}=\frac{1}{2}-\sqrt{\frac{1}{4}-\lambda}, \quad \text { and }\left|\lambda_{n}\right|<\frac{1}{4}\right\} .
$$

we define a projection

$$
P=\sum s_{n}<v_{n}, \cdot>v_{n}
$$

where

$$
s_{n}=\left\{\begin{array}{ll}
1, & \text { if } n \in N_{+}, \\
0, & \text { if } n \in N_{-} \cup N_{0} .
\end{array} .\right.
$$

Notice that $N_{0}$ is a finite set, and if $n \in N_{+} \cup N_{-}$then

$$
\left|s_{n}-\mu_{n}\right|=\left|\frac{1}{2}-\sqrt{\frac{1}{4}-\lambda_{n} \mid}<2\right| \lambda_{n} \mid .
$$

Thus $P-X \in \mathcal{K}_{\tau}(\mathfrak{H})$.

Now we are ready to apply an argument similar to that in the proof of ([B-D-F2], Theorem 4.8), also see 4.11 of [W3].

THEOREM 2.21. There is a natural isomorphism

$$
\operatorname{Ext}_{\tau}\left(\oplus_{i=1}^{n} A_{i}^{\infty}\right) \simeq \oplus_{i=1}^{n} \operatorname{Ext}_{\tau}\left(A_{i}^{\infty}\right)
$$

Proof. By induction, it suffices to consider $n=2$. For any completely positive $\tau$-almost representations

$$
\phi_{i}: A_{i} \longrightarrow \mathcal{L}(\mathfrak{H}), \quad i=1,2,
$$

let

$$
\gamma\left(\phi_{1}, \phi_{2}\right): A_{1} \oplus A_{2}\left(\dot{\phi}_{1}, \phi_{2}\right) \rightarrow \longrightarrow \mathcal{L}(\mathfrak{H}) \oplus \mathcal{L}(\mathfrak{H}) \stackrel{\zeta}{\rightarrow} \longrightarrow \mathcal{L}(\mathfrak{H})
$$

be the composition map (see 2.12). it is easy to check that $\gamma$ is defined on the unitarily equivalence classes, and that $\gamma$ maps pairs 
of degenerate extensions of $A_{1}$ and $A_{2}$ to degenerate extensions of $A_{1} \oplus A_{2}$, therefore induces a canonical group homomorphism

$$
\gamma_{*}: \operatorname{Ext}_{\tau}\left(A_{1}\right) \oplus \operatorname{Ext}_{\tau}\left(A_{2}\right) \longrightarrow \operatorname{Ext}_{\tau}\left(A_{1} \oplus A_{2}\right) .
$$

Conversely, given any completely positive $\tau$-almost representations

$$
\phi: A_{1} \oplus A_{2} \longrightarrow \mathcal{L}(\mathfrak{H})
$$

let $X_{1}=\phi(1,0)$ and $X_{2}=\phi(0,1)$. Then we have

$$
X_{1}^{2}-X_{1} \in \mathcal{K}_{\tau}(\mathfrak{H}), \quad \text { and } \quad X_{1}^{*}-X_{1} \in \mathcal{K}_{\tau}(\mathfrak{H}) .
$$

By Lemma 2.20, we can find a $\mathcal{K}_{\tau}$-perturbation $P_{1}$ of $X_{1}$ which is a projection. Let $P_{2}=I-P$. Since $\phi$ is unital, so $X_{2}-P_{2} \in \mathcal{K}_{\tau}(\mathfrak{H})$.

Let

$$
\iota_{i}: \mathcal{L}\left(P_{i} \mathfrak{H}\right) \longrightarrow \mathcal{L}(\mathfrak{H}), \quad i=1,2,
$$

be any spatial isomorphisms. For any $\phi \in \mathfrak{E x t}_{\tau}\left(A_{1} \oplus A_{2}\right)$, we define

$$
\delta([\phi])=\left(\left[\phi_{1}\right],\left[\phi_{2}\right]\right) \in \mathfrak{E x t}_{\tau}\left(A_{1}\right) \oplus \mathfrak{E x t}_{\tau}\left(A_{2}\right),
$$

where

$$
\phi_{1}\left(a_{1}\right)=\iota_{1}\left(P_{1} \phi\left(a_{1}, 0\right) P_{1}\right), \quad \forall a_{1} \in A_{1}
$$

and

$$
\phi_{1}\left(a_{2}\right)=\iota_{2}\left(P_{2} \phi\left(0, a_{2}\right) P_{2}\right), \quad \forall a_{2} \in A_{2} .
$$

It is clear that $\delta$ does not depend on particular choices of the isomorphisms $\iota_{1}$ and $\iota_{2}$. To see $\delta$ is well-defined on the unitarily equivalence class of $\phi$, it remains to verify that $\left[\phi_{1}\right]$ and $\left[\phi_{2}\right]$ are independent of the choice of $P_{1}$.

Let $P_{1}^{\prime}$ be another projection such that $P_{1}^{\prime}-X_{1} \in \mathcal{K}_{\tau}$. Then

$$
\begin{aligned}
& \iota_{1}\left(P_{1} \phi\left(a_{1}, 0\right) P_{1}\right)-\iota_{1}\left(P_{1}^{\prime} \phi\left(a_{1}, 0\right) P_{1}^{\prime}\right) \\
& \quad=\iota_{1}\left(P_{1} \phi\left(a_{1}, 0\right)\left(P_{1}-P_{1}^{\prime}\right)\right)+\iota_{1}\left(\left(P_{1}-P_{1}^{\prime}\right) \phi\left(a_{1}, 0\right) P_{1}^{\prime}\right) \in \mathcal{K}_{\tau}(\mathfrak{H}) .
\end{aligned}
$$

Similarly for $\phi_{2}$.

If $\phi$ is a representation, we may take $P_{i}=X_{i}, i=1,2$. Both $\phi_{1}$ and $\phi_{2}$ become representations. Thus

$$
\delta_{*}: \operatorname{Ext}_{\tau}\left(A_{1} \oplus A_{2}\right) \longrightarrow \operatorname{Ext}_{\tau}\left(A_{1}\right) \oplus \operatorname{Ext}_{\tau}\left(A_{2}\right)
$$


is a well-defined group homomorphism.

By construction, one verifies easily

$$
\delta \circ \gamma\left(\phi_{1}, \phi_{2}\right) \underset{\tau}{\rightarrow} \simeq\left(\phi_{1}, \phi_{2}\right), \quad \forall\left(\phi_{1}, \phi_{2}\right) \in \mathfrak{E x t}_{\tau}\left(A_{1}\right) \oplus \mathfrak{E x t}_{\tau}\left(A_{2}\right)
$$

and

$$
\gamma \circ \delta(\phi) \underset{\tau}{\rightarrow} \simeq(\phi), \quad \forall \phi \in \mathfrak{E x t}_{\tau}\left(A_{1} \oplus A_{2}\right) .
$$

Thus $\delta$ is an isomorphism.

2.22. Recall that an operator ideal $\mathcal{K}_{\tau}(\mathfrak{H})$ is given by a symmetric locally $m$-convex topology $\tau$ on $\mathcal{K}_{f}(\mathfrak{H})$, the ideal of finite rank operators. If $\tau$ is a (nonsymmetric) locally $m$-convex topology on $M_{\infty}(\mathbb{C})$, its completion $\mathcal{K}_{\tau}(\mathfrak{H})$ will no longer be an ideal of $\mathcal{L}(\mathfrak{H})$. Assume that

$$
\zeta: M_{2}(\mathcal{L}(\mathfrak{H})) \longrightarrow \mathcal{L}(\mathfrak{H})
$$

is again a spatial isomorphism taking $M_{2}\left(\mathcal{K}_{\tau}(\mathfrak{H})\right)$ onto $\mathcal{K}_{\tau}(\mathfrak{H})$, the constructions from 2.4 to 2.7 and from 2.11 to 2.15 can be generalized to this situation with the following modification (c.f. Definition 2.2 of $[\mathbf{W} 2])$ :

(1) The algebra $\mathcal{L}(\mathfrak{H})$ of bounded operators should be replaced everywhere by the algebra $\mathcal{L}_{\tau}(\mathfrak{H}):=M\left(\mathcal{K}_{\tau}(\mathfrak{H})\right) \cap \mathcal{L}(\mathfrak{H})$, where $M\left(\mathcal{K}_{\tau}(\mathfrak{H})\right)$ is the multiplier algebra of $\mathcal{K}_{\tau}(\mathfrak{H})$.

For example, in Definition 2.4, we require in addition that $\operatorname{Im}(\pi) \subset \mathcal{L}_{\tau}(\mathfrak{H})$.

(2) The unitary operators in $\mathcal{U}\left(\mathfrak{H}_{1}, \mathfrak{H}_{2}\right)$ (see 2.11) be replaced by the unitary operators in $\mathcal{U}_{\tau}\left(\mathfrak{H}_{1}, \mathfrak{H}_{2}\right)$, which are the ones preserving $\mathcal{K}_{\tau}\left(\mathfrak{H}_{i}\right), i=1,2$, under conjugacy.

Such an example is provided by the algebra $\mathcal{K}_{\infty}(\mathfrak{H})$ of smoothing operators. Fixing an orthonormal basis $\left\{e_{i}\right\}$ of $\mathfrak{H}$, these operators have matrixes with rapidly decreasing entries. The seminorms are given by

$$
\left\|\left(a_{i, j}\right)\right\|_{n}=\sum_{i, j}(1+|i|+|j|)^{n}\left|a_{i, j}\right|
$$

for $n=0,1,2, \ldots$ (cf. Lemma 3.6 below.)

A large class of such $\mathcal{K}_{\tau}$ arise in important examples of smooth extensions involving algebras of pseudodifferential operators on a compact smooth manifold with smooth symbols of various orders [W4]. 


\section{Smooth extensions of $S^{1}$ and totally disconnected spaces.}

3.1. Let $\Lambda$ be a compact subset of $\mathbb{R}$. Without loss of generality, we assume that $\Lambda \subset(0, \infty)$. Set $A=C(\Lambda)$, which has a single generator $x$. Let $\left(A^{\infty}, A\right)$ be any smooth algebra. We shall say $\left(A^{\infty}, A\right)$ is regular, if $A^{\infty}$ also has a single generator.

EXAMPle 3.2. Let $A=C[0,1]$, then the smooth algebra $\left(A^{\infty}, A\right)$ is regular whenever $A^{\infty}=C^{(k)}[0,1]$, for any $k=$ $0,1,2, \ldots, \infty$.

Proof. We check that the algebra $A_{0}^{\infty}$ of all complex polynomials in $x$ is dense in $A^{\infty}=C^{(k)}[0,1]$, for any $k=0,1,2, \ldots, \infty, \omega$. This is true for $k=0$ by Stone-Weierstrass Theorem.

Fix $f \in C^{(k)}[0,1]$ and $\epsilon>0$, we choose $p_{k} \in A_{0}^{\infty}$ such that

$$
\sup _{x \in[0,1]}\left|p_{k}(x)-f^{(k)}(x)\right|<\epsilon .
$$

Let

$$
p_{k-1}(x)=\int_{0}^{x} p_{k}(t) d t+f^{(k-1)}(0),
$$

and define $p_{k-2}, \ldots, p_{0}$ inductively. Then

$$
\left\|p_{0}-f\right\|_{C^{(k)}}=\max _{j=0, \ldots, k} \sup _{x \in[0,1]}\left|p_{0}^{(j)}(x)-f^{(j)}(x)\right| \leq \epsilon .
$$

Thus the algebra $A_{0}^{\infty}$ is dense in $A^{\infty}=C^{(k)}[0,1]$ with respect to the Banach norm $\|\cdot\|_{C^{(k)}}$. Recall that the Fréchet algebra $C^{\infty}[0,1]$ is the dense projective limit of the system

$$
\cdots \hookrightarrow C^{(k)}[0,1] \hookrightarrow C^{(k-1)}[0,1] \hookrightarrow \ldots,
$$

it follows that $A_{0}^{\infty}$ is also dense in $C^{\infty}[0,1]$.

LEMma 3.3. Let $\Lambda$ be a compact subset of $\mathbb{R}$. Let $\left(A^{\infty}, A\right)$ be a regular smooth algebra with $A=C(\Lambda)$. Then $\operatorname{Ext}_{s, \tau}\left(A^{\infty}\right)=0$.

Proof. The algebra $\left(A^{\infty}, A\right)$ is generated by a single generater $Z$. Thus a $\tau$-smooth extension $\phi$ of $\left(A^{\infty}, A\right)$ is uniquely determined by the operator $T=\phi(x)$. Any operator $T$ with $\sigma_{e}(T)=\Lambda$ and $T-T^{*} \in \mathcal{K}_{\tau}$ defines a $\tau$-smooth extension $\phi$. The extension $\phi$ is degenerated if $\phi(x)$ is self-adjoint. 
A completely positive $\tau$-almost representation $\phi^{\prime}$ of $\left(A^{\infty}, A\right)$ is uniquely determined by $\phi^{\prime}(x):=\frac{1}{2}\left(T+T^{*}\right)$. Because $\phi$ is $\tau$-smooth as a completely positive map, and $\left(\phi-\phi^{\prime}\right) A_{0}^{\infty} \subset \mathcal{K}_{\tau}$, where $A_{0}^{\infty}$ is the subalgebra of all complex polynomials in $x$, we have $\left(\phi-\phi^{\prime}\right) A^{\infty} \subset$ $\mathcal{K}_{\tau}$. Thus $\phi^{\prime}$ is also a $\tau$-smooth extension of $\left(A^{\infty}, A\right)$ and unitarily equivalent to $\phi$.

The extension $\phi^{\prime}$ is degenerated because $\phi^{\prime}(x)$ is self-adjoint. Thus one concludes that

$$
\mathfrak{E x t}_{s, \tau}\left(A^{\infty}\right) \subset \mathfrak{D x t}_{s, \tau}\left(A^{\infty}\right) \text {. }
$$

Hence $\operatorname{Ext}_{s, \tau}\left(A^{\infty}\right)=0$.

Now consider a compact, second countable, totally disconnected space $X . A:=C(X)$ is generated by countably many orthogonal projections $p_{n}$. By a result of von Neumann, the positive function

$$
g:=\sum_{n} \frac{2^{n} p_{n}+1}{3^{n}}
$$

generates $A$. Thus $X$ is homeomorphic to $\Lambda:=\operatorname{Image}(g) \subset \mathbb{R}^{+}$. Let $\left(A^{\infty}, A\right)$ be a smooth algebra with $A=C(X)$, we also say $\left(A^{\infty}, A\right)$ is regular if $A^{\infty}$ is regular subalgebra under the isomorphism $\mathcal{C}(X) \simeq$ $C(\Lambda)$. Thus we can use Lemma 3.3. (c.f. the proof of Theorem 1.15 of $[\mathrm{B}-\mathrm{D}-\mathrm{F} 2])$.

Corollary 3.4. Let $X$ be a compact, second countable, totally disconnected space. Let $\left(A^{\infty}, A\right)$ be a regular smooth algebra with $A=C(X)$. Then $\operatorname{Ext}_{s, \tau}\left(A^{\infty}\right)=0$.

3.5. If $\phi$ defines a $\tau$-smooth extension, then $\phi$ also defines a $\tau$-smooth extension for any $\tau^{\prime}, \mathcal{K}_{\tau^{\prime}} \subset \mathcal{K}_{\tau}$.

Theorem 3.7 and the following fact are the key steps leading to the other main theorem (Theorem 3.8) in this section.

LEMMA 3.6. The canonical homomorphism $\alpha_{\tau}$ from $\operatorname{Ext}_{\tau} C^{\infty}\left(S^{1}\right)$ to the $B-D-F$ functor $\operatorname{Ext} C\left(S^{1}\right)$ is surjective whenever $\mathcal{K}_{\tau} \supset \mathcal{K}_{\infty}$.

Proof. We only need to show that the Toeplitz extension given by the unilateral shift is $\mathcal{K}_{\tau}$-smooth, because it is a generator for the cyclic group $\operatorname{Ext} C\left(S^{1}\right)$. 
Take any $f \in C^{\infty}\left(S^{1}\right)$. Let

$$
f=\sum_{n \in \mathbb{Z}} a_{n} e^{2 \pi n i \theta} \in C^{\infty}\left(S^{1}\right),
$$

where $\left(a_{n}\right)$ is a rapidly decreasing sequence. Let $P$ be the projection of the Hilbert space $H:=L^{2}\left(S^{1}, \mu\right)$ onto the Hardy space $\mathfrak{H}:=$ $H^{2}\left(S^{1}, \mu\right)$, where $\mu$ is the Lebesgue measure. It is enough to verify that

$$
\left[P, M_{f}\right] \in \mathcal{K}_{\infty}(H), \quad \forall f \in C^{\infty}\left(S^{1}\right) .
$$

With respect to the standard basis $\left\{e_{n}\right\}$ of $H$, where $e_{n}=e^{2 \pi n i \theta}$, the operator

$$
\begin{aligned}
{\left[P, M_{f}\right] } & =\sum_{i=1}^{\infty} \sum_{k=0}^{i-1} a_{i}<e_{k}, .>e_{i-k}+ \\
& -\sum_{i=-1}^{-\infty} \sum_{k=0}^{i-1} a_{i}<e_{-k}, .>e_{i+k}
\end{aligned}
$$

takes form

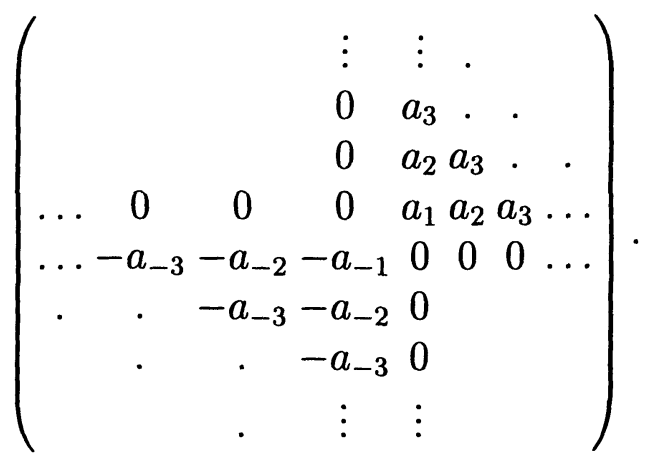

Because

$$
(i+j)^{n} a_{i+j} \longrightarrow 0 \text { as } i+j \rightarrow \infty, i, j>0
$$

and

$$
(i+j)^{n} a_{-i-j} \longrightarrow 0 \text { as } i+j \rightarrow \infty, i, j>0,
$$

(1) is a Schwartz matrix. So $\left[P, M_{f}\right] \in \mathcal{K}_{\infty} \subset \mathcal{K}_{\tau}$ for all $f \inf C^{\infty}\left(S^{\top}\right)$. It follows that the Toeplitz extension $\left(P, M\right.$. is $\mathcal{K}_{\tau}$-smooth.

THEOREM 3.7. Let $\mathcal{K}_{\tau}$ be any operator ideal (1.4). Let $\phi$ be any $\mathcal{K}_{\tau}$-smooth extension of $\left(C^{\infty}\left(S^{1}\right), C\left(S^{1}\right)\right)$. Then $\phi$ is $\tau$-smooth as 
a completely positive map. The smooth operator algebra $E^{\infty}$ given by the extension $\phi($ see 2.7$)$ is $\mathcal{K}_{\tau}$-smooth $\bmod \mathcal{K}_{\tau}$.

Proof. By Proposition 2.16, it suffices to show that the completely positive map $\phi$ is $\mathcal{K}_{\tau}$-smooth.

Let $\phi_{0}:\left(E^{\infty}, E\right) \longrightarrow \mathcal{L}(\mathfrak{H})$ be another completely positive map such that

$$
\left(\phi-\phi_{0}\right) f \in \mathcal{K}_{\tau}(\mathfrak{H}), \quad \text { for } \forall f \in A_{0}^{\infty},
$$

where $A_{0}^{\infty}$ is the algebra of all Laurent polynomials in $z$. We need to show that

$$
\phi(f)-\phi_{0}(f) \in \mathcal{K}_{\tau}(\mathfrak{H}), \quad \text { for all } f \in C^{\infty}\left(S^{1}\right) .
$$

The Fourier expansion of $f$ has form

$$
f=\sum_{n \in \mathbb{Z}} a_{n} z^{n}
$$

where $\left(a_{n}\right)$ is rapidly decay. Denote

$$
T:=\phi(z) \text { and } T_{0}:=\phi_{0}(z) .
$$

The assertion (2) will follow from (4), (5) and (6) below:

$$
\phi(f)-\sum_{j=0}^{\infty} a_{j} T^{j}+\sum_{j=1}^{\infty} a_{-j} T^{* j} \in \mathcal{K}_{\tau}(\mathfrak{H})
$$

$$
\phi_{0}(f)-\sum_{j=0}^{\infty} a_{j} T_{0}^{j}+\sum_{j=1}^{\infty} a_{-j} T_{0}^{* j} \in \mathcal{K}_{\tau}(\mathfrak{H})
$$

$$
\sum_{j=0}^{\infty} a_{j} T^{j}+\sum_{j=1}^{\infty} a_{-j} T^{* j}-\sum_{j=0}^{\infty} a_{j} T_{0}^{j}-\sum_{j=1}^{\infty} a_{-j} T_{0}^{* j} \in \mathcal{K}_{\tau}(\mathfrak{H})
$$

We will verify (6), then (5). The estimate also establishes (4). Fix any seminorm $p_{m}$ (see 1.5). Because

$$
p_{m}\left(T^{n}-T_{0}^{n}\right) \leq \sum_{i=1}^{n} p_{m}\left(T_{0}^{n-i}\left(T-T_{0}\right) T^{i-1}\right) \leq n p_{m}\left(T-T_{0}\right)
$$


notice $\|T\| \leq 1$ and $\left\|T_{0}\right\| \leq 1$, we obtain

$$
\sum_{n=0}^{\infty}\left|a_{n}\right| p_{m}\left(T^{n}-T_{0}^{n}\right) \leq \sum_{n=0}^{\infty} n\left|a_{n}\right| p_{m}\left(T-T_{0}\right)<\infty .
$$

Similarly

$$
\sum_{n=0}^{\infty}\left|a_{n}\right| p_{m}\left(T^{* n}-T_{0}^{* n}\right) \leq \sum_{n=0}^{\infty} n\left|a_{n}\right| p_{m}\left(T^{*}-T_{0}^{*}\right)<\infty .
$$

This verifies (6).

Since $\phi_{0}$ is a unital completely positive map, by Stinespring's theorem, there is a ${ }^{*}$-representation $\pi$ of $C\left(S^{1}\right)$ on a Hilbert space $H$ and a projection $P$ in $H$ (we may assume the range of $P$ is $\mathfrak{H}$, because of (2)) such that

$$
\phi_{0}(f)=P \pi(f) P, \quad \forall f \in C\left(S^{1}\right) .
$$

Let $U=\pi(z)$ and denote

$$
k:=P U(1-P), \quad \tilde{k}:=(1-P) U P .
$$

Then by the assumption (1), we see

$$
\begin{aligned}
k \tilde{k} & =P U(1-P) U P=\phi_{0}\left(z^{2}\right)-\phi_{0}(z)^{2} \in \mathcal{K}_{\tau}(\mathfrak{H}), \\
k k^{*} & =P U(1-P) U^{*} P=1-\phi_{0}(z) \phi_{0}(z)^{*} \in \mathcal{K}_{\tau}(\mathfrak{H}), \\
\tilde{k}^{*} \tilde{k} & =P U^{*}(1-P) U P=1-\phi_{0}(z)^{*} \phi_{0}(z) \in \mathcal{K}_{\tau}(\mathfrak{H}) .
\end{aligned}
$$

Thus $k, \tilde{k} \in \mathcal{K}_{\tau}^{\frac{1}{2}}(H)$ (see 1.5), and

$$
\tilde{k}-k=[U, P] \in \mathcal{K}_{\tau}^{\frac{1}{2}}(H) .
$$

Now notice

$$
\begin{aligned}
\phi_{0}\left(z^{j+1}\right)-\phi_{0}(z) \phi_{0}\left(z^{j}\right) & =P U(1-P) U^{j} P \\
& =P U(1-P) U^{j-1}([U, P]+P U) .
\end{aligned}
$$

By a Schwarz inequality, we have

$$
\begin{aligned}
& p_{m}\left(\phi_{0}\left(z^{j+1}\right)-\phi_{0}(z) \phi_{0}\left(z^{j}\right)\right) \\
& \quad \leq p_{m}\left(P U(1-P) U^{j-1}[U, P]\right)+p_{m}\left(P U(1-P) U^{j-1} P U\right) \\
& \quad \leq p_{m}^{2}(k) p_{m}^{2}(\tilde{k}-k)+p_{m}\left(\phi_{0}\left(z^{j}\right)-\phi_{0}(z) \phi_{0}\left(z^{j-1}\right)\right) .
\end{aligned}
$$


By induction we get

$$
\begin{aligned}
& p_{m}\left(\phi_{0}\left(z^{j+1}\right)-\phi_{0}(z) \phi_{0}\left(z^{j}\right)\right) \\
& \quad \leq(j-1) p_{m}^{2}(k) p_{m}^{2}(\tilde{k}-k)+p_{m}^{2}(k) p_{m}^{2}(\tilde{k}) \\
& \quad \leq(j-1)\left(p_{m}^{2}(k)+p_{m}^{2}(\tilde{k}-k)\right)^{2} .
\end{aligned}
$$

It follows that

$$
\begin{aligned}
& p_{m}\left(\phi_{0}\left(z^{n}\right)-T_{0}^{n}\right) \\
& \leq \sum_{j=1}^{n-1} p_{m}\left(T_{0}^{n-j-1}\left(\phi_{0}\left(z^{j+1}\right)-T_{0} \phi_{0}\left(z^{j}\right)\right)\right) \\
& \leq \sum_{j=1}^{n-1} p_{m}\left(\phi_{0}\left(z^{j+1}\right)-T_{0} \phi_{0}\left(z^{j}\right)\right) \\
& \leq \sum_{j=1}^{n-1}(j-1)\left(p_{m}^{2}(k)+p_{m}^{2}(\tilde{k}-k)\right)^{2} \\
& \quad=\frac{(n-1)(n-2)}{2}\left(p_{m}^{2}(k)+p_{m}^{2}(\tilde{k}-k)\right)^{2} .
\end{aligned}
$$

Consequently, we have

$$
\text { (9) } \begin{aligned}
\sum_{j=0}^{\infty}\left|a_{n}\right| p_{m}\left(\phi_{0}\left(z^{n}\right)-T_{0}^{n}\right) \\
\leq \sum_{n=0}^{\infty}\left|a_{n}\right| \frac{(n-1)(n-2)}{2}\left(p_{m}^{2}(k)+p_{m}^{2}(\tilde{k}-k)\right)^{2}<\infty
\end{aligned}
$$

and

$$
\text { (10) } \begin{aligned}
& \sum_{j=0}^{\infty}\left|a_{-n}\right| p_{m}\left(\phi_{0}\left(z^{-n}\right)-T_{0}^{* n}\right) \\
& \quad \leq \sum_{n=0}^{\infty}\left|a_{-n}\right| \frac{(n-1)(n-2)}{2}\left(p_{m}^{2}(k)+p_{m}^{2}(\tilde{k}-k)\right)^{2}<\infty .
\end{aligned}
$$

By the $C^{*}$-norm continuity and linearity of $\phi_{0}$, we have

$$
\phi_{0}(f)=\sum_{j \in \mathbb{Z}} a_{n} \phi_{0}\left(z^{n}\right) \in \mathcal{L}(\mathfrak{H}) .
$$

From (9), (10) and (11), we get (5). Therefore $\phi$ is $\tau$-smooth as a complete positive map. 
TheOREM 3.8. For any Fréchet operator ideal, we have

$$
\operatorname{Ext}_{\tau} C^{\infty}\left(S^{1}\right) \simeq \operatorname{Ext} C\left(S^{1}\right) \simeq \mathbb{Z}
$$

Proof. We would like to thank N.C. Phillips for a discussion which simplified the argument. By Weyl-von Neumann-Berg Theorem,

$$
\operatorname{Ext} C\left(S^{1}\right) \simeq \operatorname{Ext} C\left(S^{1}\right) .
$$

A generator of $\operatorname{Ext} C\left(S^{1}\right) \simeq \mathbb{Z}$ is the Toeplitz extension associated with the unilateral shift of index -1 . Lemma 3.6 says that the canonical map

$$
\alpha_{\tau}: \operatorname{Ext}_{\tau} C^{\infty}\left(S^{1}\right) \longrightarrow \operatorname{Ext} C\left(S^{1}\right)
$$

is surjective. It remains to show that it is injective.

Let $[\phi] \in \mathfrak{E x t}_{\tau} C^{\infty}\left(S^{1}\right), V=\phi(z)$, so that

$$
V V^{*}-1, V^{*} V-1 \in \mathcal{K}_{\tau} \text {. }
$$

Assume that $\alpha[\phi] \in \mathfrak{D} \mathfrak{x t C}\left(S^{1}\right)$, thus Index $V=0$. Therefore a finite rank perturbation $W$ of $V$ is invertible.

Let $W=U|W|$ be the polar decomposition of $W,|W|=$ $\left(W^{*} W\right)^{1 / 2}>0$ is invertible, and $U$ is unitary.

It is easy to see

$$
|W|-1=\left(W^{*} W-1\right)(|W|+1)^{-1} \in \mathcal{K}_{\tau},
$$

thus

$$
W-U=W(|W|-1)|W|^{-1} \in \mathcal{K}_{\tau} .
$$

By Theorem 3.7, any completely positive map

$$
\phi^{\prime}:\left(C^{\infty}\left(S^{1}\right), C\left(S^{1}\right)\right) \longrightarrow \mathcal{L}(\mathfrak{H})
$$

with $\phi(z)-\phi^{\prime}(z) \in \mathcal{K}_{\tau}(\mathfrak{H})$ will define a $\mathcal{K}_{\tau}$-smooth extension unitarily equivalent to $\phi$.

Define a $\tau$-smooth extension $\rho$ of $C^{\infty}\left(S^{1}\right)$ by

$$
\rho(z)=U, \quad \rho(\bar{z})=U^{*},
$$

then

$$
U-V=(U-W)+(W-V) \in \mathcal{K}_{\tau}(\mathfrak{H})
$$


so $\rho \underset{\tau}{\rightarrow} \sim \phi$. Since $\rho$ is degenerated, we deduce that $[\phi] \in \mathfrak{D x t}_{\tau} C^{\infty}\left(S^{1}\right)$.

It is well-known that a Frechet algebra has homological functional calculus, but in general not $C^{\infty}$-functional calculus. The cruxial step in the proof above is precisely the $\tau$-smoothness of $C^{\infty}\left(S^{1}\right)$ (Theorem 3.7), thereby $\rho(z)=U$ defines uniquely a $\tau$-smooth extension $\rho$.

\section{REFERENCES}

[Ar] W. Arveson, Notes on extensions of $C^{*}$-algebras, Duke Math J., 44 No. 2 (1977), 329-355.

[At] M. F. Atiyah, Global theory of elliptic operators, Proc. Int. Conf. on Fun. Ana. and Related Topics (1970), 21-23, Univ. Tokyo Press, Tokyo.

[B-C] B. Blackadar and J. Cuntz, Differential Banach algebra norms and smooth subalgebras of $C^{*}$-algebras, preprint.

[Br] L. Brown, The Determinant Invariant for Operators with Trace Class Self-Commutators, Lecture Notes of Math., 345 (1973), 210-228, SpringerVerlag.

[B-D-F1] L. Brown, R. Douglas and P. Fillmore, Extensions of $C^{*}$-Algebras and K-Homology, Lecture Notes of Math., 345 (1973), Springer-Verlag.

[B-D-F2] , Extensions of $C^{*}$-algebras and K-homology, Ann. Math., (2), 105 (1977), 265-324.

[C-P] R. Carey and J.D. Pincus Almost Commuting Algebras, K-Theory and Operator Algebras, Lecture Notes of Math., 575 (1977), Springer-Verlag.

[C] A. Connes, Noncommutative Differential Geometry I, II, Publ. I.H.E.S., 62 (1986), 257-360.

[C-K] A. Connes and M. Karoubi Caractèr multiplicatif d'un module de Fredholm, C. R. Acad. Sci. Paris Sér. I Math., 299 (1984), 963-968.

[D] R. Douglas, On the Smoothness of Elements of Ext, Topics in Modern Operator Theory, Birkhauser, 1981, 63-69.

[D-V] R. Douglas and D. Voiculescu, On the smoothness of sphere extensions, J. Operator Theory.

[E] E. Effros, Advances in quantized functional calculus, Proc. I.C.M. (1986), 906-916.

[G] G. Gong, Smooth extensions for a finite $C W$-complex, Bull. Amer. Math. Soc., 22 (Jan. 1990), 73-78.

[H-H] J. W. Helton and R. Howe Integral Operators: Commutators, Traces, Index, and Homology, Lecture Notes of Math., 345 (1973), 141-209, Springer-Verlag.

[K] G. Kasparov, The operator K-functor and extensions of $C^{*}$-algebras, Izv. 
Akad. Nauk SSSR, Ser. Math., 44 (1980), 571-636.

[Ky] Ky Fan, Maximal properties and inequalities for the eigenvalues of completely continuous operators, Proc. Nat. Acad. Sci. USA, 37 (1951), 760-766.

[P] N.C. Phillips, K-theory for Fréchet algebras, Internat. J. Math., (1991).

[R] M. Rieffel,Deformation quantization of Heisenberg manifolds, Comm. Math. Phy., 122 (1989), 531-562.

[Sl] N. Salinas, Smooth extensions and smooth joint quasitriangularity, Operator Theory: Adv. Appl., 11 (1983), 303-332.

[Sw] L.B. Schweitzer, $A$ short proof that $M_{n}(A)$ is local if $A$ is local and Fréchet, Internat. J. Math., 3 (1992), 581-589.

[V2] D. Voiculescu, Some results on norm-ideal perturbations of Hilbert space operators, J. Operator Theory, 2 (1979), 3-37; II, ibid 5 (1981), 77-100.

[W1] X. Wang Bivariant Chern Character II, Canad. J. Math., 45 (May 1992), 400-435.

[W2] - KK-theories for topological algebras, K-theory, 5 (1991), 97-150.

[W3] - Theorems of Voiculescu and Stinespring; Extensions of Smooth Algebras yr, J. Funct. Anal., (to appear).

[W4] - Quantizations and noncommutative geometry, to appear.

[W5] - Geometric BRST quantization and smooth Toeplitz extensions, in preparation.

[W6] - Voiculescu Theorem, Sobolev Lemma; and extensions of smooth algebras, Bull. Amer. Math. Soc., 27 (Oct. 1992), 292-297.

[W7] _ Smooth K-homology, Chern character and a noncommutative Sobolev Lemma, preprint.

[W8] - Dirac operators on the tori; and Gauss' class groups, preprint.

[W9] _ Spherical spinors, orthogonal polynomials and extensions, in preparation.

Received July 30, 1992 and in revised form May 1, 1993. This research was supported in part by National Science Foundation grant DMS 9012753.

\section{NYU-COURANT}

NEw YORK, NEw YORK 10021

E-mail address: xlwang@cims.nyu.edu. 



\title{
PACIFIC JOURNAL OF MATHEMATICS
}

Founded by E. F. Beckenbach (1906-1982) and F. Wolf (1904-1989)

\section{EDITORS}

Sun-Yung Alice Chang (Managing Editor)

University of California

Los Angeles, CA 90095-1555

pacific@math.ucla.edu

F. Michael Christ

University of California

Los Angeles, CA 90095-1555

christ@math.ucla.edu

\section{Thomas Enright}

University of California

San Diego, La Jolla, CA 92093

tenright@ucsd.edu

Nicholas Ercolani

University of Arizona

Tucson, AZ 85721

ercolani@math.arizona.edu
Robert Finn

Stanford University

Stanford, CA 94305

finn@gauss.stanford.edu

Vaughan F. R. Jones

University of California

Berkeley, CA 94720

vfr@math.berkeley.edu

Steven Kerckhoff

Stanford University

Stanford, CA 94305

spk@gauss.stanford.edu
Martin Scharlemann

University of California

Santa Barbara, CA 93106 mgscharl@math.ucsb.edu

Gang Tian

Courant Institute

New York University

New York, NY 10012-1100

tiang@taotao.cims.nyu.edu

V. S. Varadarajan

University of California

Los Angeles, CA 90095-1555

vsv@math.ucla.edu

\section{SUPPORTING INSTITUTIONS}

\section{CALIFORNIA INSTITUTE OF TECHNOLOGY NEW MEXICO STATE UNIVERSITY \\ OREGON STATE UNIVERSITY \\ STANFORD UNIVERSITY \\ UNIVERSITY OF ARIZONA \\ UNIVERSITY OF BRITISH COLUMBIA \\ UNIVERSITY OF CALIFORNIA \\ UNIVERSITY OF HAWAII}

\author{
UNIVERSITY OF MONTANA \\ UNIVERSITY OF NEVADA, RENO \\ UNIVERSITY OF OREGON \\ UNIVERSITY OF SOUTHERN CALIFORNIA \\ UNIVERSITY OF UTAH \\ UNIVERSITY OF WASHINGTON \\ WASHINGTON STATE UNIVERSITY
}

The supporting Institutions listed above contribute to the cost of publication of this Journal, but they are not owners or publishers and have no responsibility for its contents or policies.

Manuscripts must be prepared in accordance with the instructions provided on the inside back cover.

The Pacific Journal of Mathematics (ISSN 0030-8730) is published monthly except for July and August. Regular subscription rate: $\$ 215.00$ a year (10 issues). Special rate: $\$ 108.00$ a year to individual members of supporting institutions.

Subscriptions, orders for back issues published within the last three years, and changes of subscribers address should be sent to Pacific Journal of Mathematics, P.O. Box 4163, Berkeley, CA 94704-0163, U.S.A. Prior back issues are obtainable from Kraus Periodicals Co., Route 100, Millwood, NY 10546.

The Pacific Journal of Mathematics at the University of California, c/o Department of Mathematics, 981 Evans Hall Berkeley, CA 94720 (ISSN 0030-8730) is published monthly except for July and August. Second-class postage paid at Berkeley, CA 94704, and additional mailing offices. POSTMASTER: send address changes to Pacific Journal of Mathematics, P.O. Box 6143, Berkeley, CA 94704-0163.

\author{
PUBLISHED BY PACIFIC JOURNAL OF MATHEMATICS at University of California, \\ Berkeley, CA 94720, A NON-PROFIT CORPORATION \\ This publication was typeset using AMS-LATEX, \\ the American Mathematical Society's TEX macro system. \\ Copyright (C) 1995 by Pacific Journal of Mathematics
}




\section{PACIFIC JOURNAL OF MATHEMATICS}

Volume $169 \quad$ No. $2 \quad$ June 1995

On Banach spaces $Y$ for which $B(C(\Omega), Y)=K(C(\Omega), Y)$

201

SHAMIM ISMAIL ANSARI

Convergence of infinite exponentials

219

GENNADY BACHMAN

Cohomologie d'intersection modérée. Un théorème de de Rham

235

Bohumil Cenkl, Gilbert Hector and Martintxo

SARALEGI-ARANGUREN

Kleinian groups with an invariant Jordan curve: $J$-groups

291

Ruben A. Hidalgo

Multiplicative functions on free groups and irreducible representations 311

M. GABRiella KuHN and Tim STEGer

A Diophantine equation concerning finite groups

MAOHUA LE

Nilpotent characters

343

GABRIEL NAVARRO

Smooth extensions and quantized Fréchet algebras

XiaOlU WANG 